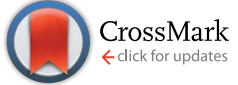

Cite this: RSC Adv., 2017, 7, 5528

Received 2nd December 2016

Accepted 5th January 2017

DOI: 10.1039/c6ra27680f

www.rsc.org/advances

\section{Hybrid extracellular matrix microspheres for development of complex multicellular architectures $\dagger$}

\author{
Christine Affolter-Zbaraszczuk, $\dot{t}^{\mathrm{a}}$ Hayriye Ozcelik, $t^{\mathrm{b}}$ Florent Meyer, ${ }^{\text {ab }}$ Olivier Gallet, ${ }^{\mathrm{c}}$ \\ Philippe Lavalle, ${ }^{\mathrm{ab}}$ Vincent Ball, ${ }^{\mathrm{ab}}$ Camélia Matei Ghimbeu, ${ }^{d}$ Pierre Schaaf ${ }^{\star a b e}$ \\ and Helena Knopf-Marques ${ }^{\text {ab }}$
}

This study aims to develop a system for tissue engineering or wound management using the advantages of ECM components like gelatin, hyaluronic acid, fibronectin. We developed for the first time a hybrid $\mathrm{CaCO}_{3}$ microparticular system for creating a building block strategy. This promising method is easy to apply, versatile and will allow the buildup of any kind of targeted tissues.
The development of organs and tissues by tissue engineering methods involves careful control of cell organization to achieve full functionality. The extracellular matrix is a complex network based on glycosaminoglycans (such as hyaluronic acid) and proteins (such as collagen) secreted by cells. This complex network forms a supporting framework that is responsible for holding cells and tissues together. ${ }^{1}$

The design of scaffolds mimicking ECM both from a chemical and a mechanical point of view is a way to direct cell behavior. ${ }^{2}$ Microporous or mesoporous scaffolds are promising architectures for tissue engineering constructs to direct cell attachment and differentiation ${ }^{3}$ and also for local release of biological cues at the implantation site. ${ }^{4}$ Typically, porous materials are prepared from emulsions, by solvent evaporation or by precipitation techniques. Alternatively, templating techniques $^{5}$ are used when precise control over material properties, like composition, morphology and porosity, is desired.

Hydrogel microparticles that mimic the ECM are extensively used for cell encapsulation, culture and transplantation. ${ }^{6}$ Recently, Lu and colleagues used complex hydrogel microparticles with controlled ECM internal compartments for studying the cell-cell interactions such as cell segregations under 3D confined space and the supporting role of stromal cells in

aINSERM UMR 1121, 11 rue Humann, 67085 Strasbourg, France. E-mail: pierre. schaaf@inserm.fr

${ }^{b}$ Faculté de Chirurgie Dentaire, Fédération de Médecine Translationnelle de Strabourg, Université de Strasbourg, 3 rue Sainte Elisabeth, 67000 Strasbourg, France

${ }^{c}$ Errmece, E.A. 1391, Université de Cergy Pontoise, Rue Descartes, 95031 Neuville sur Oise, Cedex, France

${ }^{d}$ Institut de Science des Matériaux de Mulhouse, IS2M - CNRS UMR 7361 - UHA 15, rue Jean Starcky, 68057 Mulhouse Cedex, France

${ }^{e}$ Institut Charles Sadron, CNRS UPR 22, 23 rue du Loss, 67034 Strasbourg, France $\dagger$ Electronic supplementary information (ESI) available: Materials and methods. See DOI: $10.1039 / \mathrm{c} 6 \mathrm{ra} 27680 \mathrm{f}$

\$ These authors contributed equally. maintaining in vitro culture of hepatocytes. It was demonstrated the possibility of combining those uniform microparticles with addressable micro-well systems for potential reconfigurable paracrine cell co-culture applications. ${ }^{7}$

Other teams used $\mathrm{CaCO}_{3}$ microparticles as drug carriers for their capability to load large amounts of molecules due to the large specific surface area of the structures. ${ }^{8,9}$ For this reason, $\mathrm{CaCO}_{3}$ microparticles have been widely used as template for adsorption of substance of interest. ${ }^{10}$ Further, the surface of porous $\mathrm{CaCO}_{3}$ microparticles can be easily modified by the layer-by-layer (L-b-L) method ${ }^{9}$ or by a simple protein adsorption on the surface.

Additionally, the casting strategy is widely used to produce uniform and stable protein encapsulation by copying the structure of $\mathrm{CaCO}_{3}$ template. ${ }^{10}$ In 2012, Wang and colleagues obtained microgels by the EDTA treatment of $\mathrm{CaCO}_{3}$-gelatin particles crosslinked with genipin. Those microgels were found biocompatible and efficient carriers of bioactive agents. ${ }^{10}$ The work developed by $\mathrm{Du}$ et al. (2013) proposed the inorganic/ organic hybrid $\mathrm{CaCO}_{3}$ microparticles doped with sodium pol$\mathrm{y}$ (styrenesulfonate) as a smart drug release system. These microparticles were coated by the LbL method using aliphatic poly(urethane-amine) and sodium poly(styrene sulfonate). As a drug model, the anti-cancer doxorubicin hydrochloride (DOX) drug was selected. ${ }^{9}$

In the present study we develop a building block system based on $\mathrm{CaCO}_{3}$ microparticles doped with gelatin, hyaluronic acid and fibronectin that is suitable for creating well-defined 3D architectures. These building blocks will allow step-by-step buildup to produce stratified 3D cellularized structures. We have designed a new coating based on hybrid microparticles mimicking the extracellular matrix deposited with fibroblasts. The method is easy to apply, versatile and will allow the buildup of any kind of targeted tissues. 


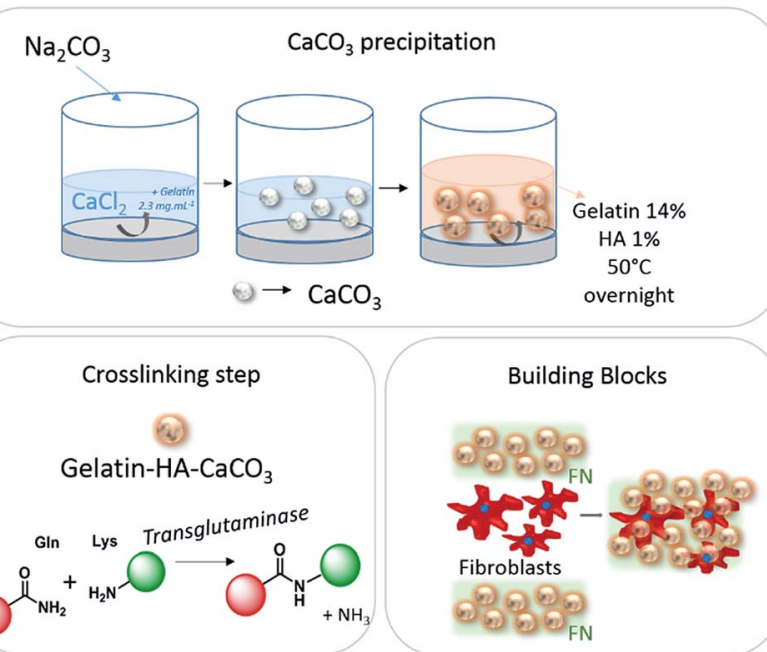

Scheme 1 Preparation of hybrid porous microparticles composed of gelatin, hyaluronic acid and $\mathrm{CaCO}_{3}$; gelatin crosslinking step and building blocks system with fibroblasts.

The preparation of the system composed of gelatin, hyaluronic acid, $\mathrm{CaCO}_{3}$ and fibronectin is shown in Scheme 1. Hyaluronic acid is a well-known major component of ECM and is present in all human tissues. ${ }^{11}$ Gelatin is a denatured, biodegradable and biocompatible protein obtained by acid and alkaline processing of collagen. ${ }^{12}$ As gelatin is soluble in aqueous solution at $37{ }^{\circ} \mathrm{C}$, its use for biomedical applications
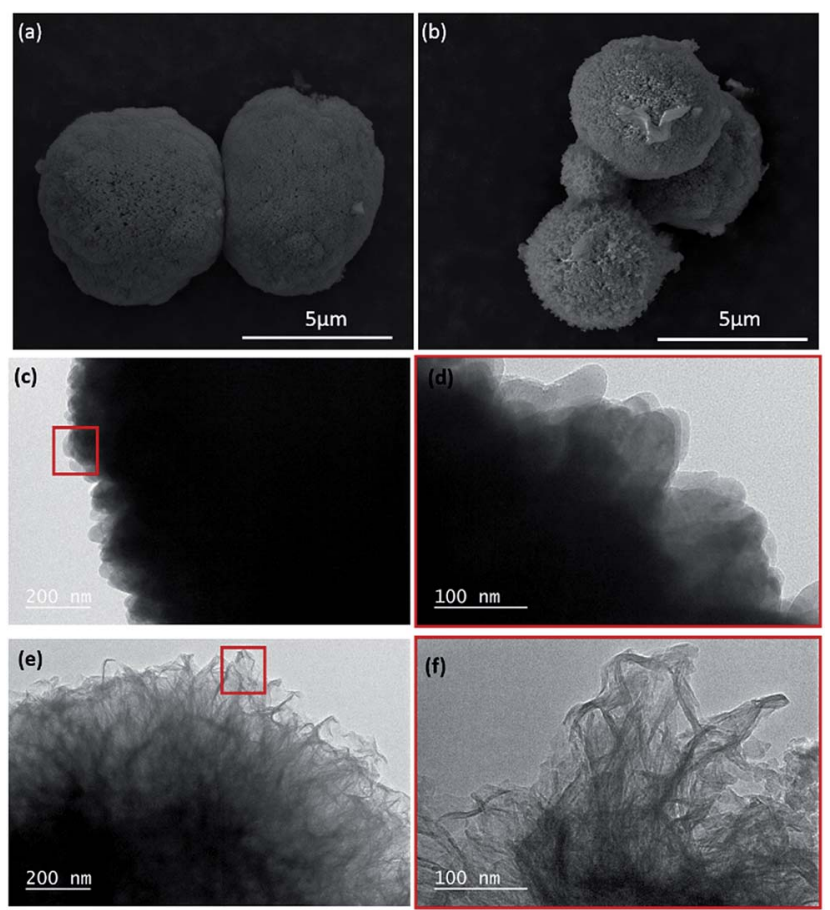

Fig. 1 Scanning electron microscopy (SEM) micrographs of particles: (a) $\mathrm{CaCO}_{3}$ and (b) gelatin- $\mathrm{HA}-\mathrm{CaCO}_{3}$ after crosslinking with transglutaminase. Transmission electron microscopy (TEM) micrographs of particles: (c) and (d) $\mathrm{CaCO}_{3}$ and (e) and (f) gelatin- $\mathrm{HA}-\mathrm{CaCO}_{3}$ after crosslinking with transglutaminase. relies on improvement of its mechanical and thermal stability by crosslinking with transglutaminase. Spherical microparticles of $\mathrm{CaCO}_{3}$ were prepared by wet chemical process in the presence of gelatin type A. ${ }^{10}$ This procedure results in spherical $\mathrm{CaCO}_{3}$ microparticles with an average diameter of $2.9 \pm 0.5 \mu \mathrm{m}$ (Fig. 1a). An additional coating with gelatin and $\mathrm{HA}$ is performed by casting strategy (Fig. 1b).

The $\mathrm{CaCO}_{3}$ and gelatin-HA-CaCO${ }_{3}$ microparticles crosslinked with transglutaminase were analyzed by transmission electron microscopy. The surface of $\mathrm{CaCO}_{3}$ microparticles formed by precipitation presents a typical crystal with a vateritic morphology, (Fig. 1c-d) which is advantageous for applications in the field of biomedical area (regenerative medicine and tissue engineering), due to its high solubility in water and biological inertness. ${ }^{13}$ While, once those particles were incubated in a hot aqueous gelatin-HA solution at $50{ }^{\circ} \mathrm{C}$ for 12 hours, a fibrous structure is observed on the surface of the particles, indicating the presence of a gelatin shell on their surface (Fig. 1e-f).

The thermogravimetric analyses (TGA) of the $\mathrm{CaCO}_{3}$ and gelatin-HA-CaCO${ }_{3}$ microparticles, show a mass loss of $1 \mathrm{wt} \%$ and $4 \mathrm{wt} \%$ between 200 and $500{ }^{\circ} \mathrm{C}$, respectively (Fig. 2a). These mass losses correspond to the decomposition of gelatin and HA, indicating that the amount of gelatin after the first step of $\mathrm{CaCO}_{3}$ microparticle formation was around $1 \mathrm{wt} \%$, whereas after the gelatin casting step its amount was about $4 \mathrm{wt} \%$. The sharpest weight loss observed between 650 and $800{ }^{\circ} \mathrm{C}$ corresponds to the decomposition of $\mathrm{CaCO}_{3}$ microparticles.

The FTIR spectra of the $\mathrm{CaCO}_{3}$ microparticles (Fig. 2b) present adsorption bands at 1440,870 and $712 \mathrm{~cm}^{-1}$ that are characteristic of $\mathrm{CaCO}_{3}$ which are assigned to the characteristic bond vibrations: $\nu 3, \nu 2$ and $\nu 4$, respectively. ${ }^{9}$ After the gelatinHA casting one observes an adsorption band at $1650 \mathrm{~cm}^{-1}$ characteristic of amide I vibrations from proteins and thus corresponding to the adsorption of gelatin in the microparticles. Although the HA presence was not detected by FTIR, it was noticed by confocal analysis, as the HA was labelled with FITC.

XRD patterns of the $\mathrm{CaCO}_{3}$ and gelatin-HA-CaCO${ }_{3}$ microparticles after crosslinking with transglutaminase confirmed the major presence of vaterite (Fig. 2c). Two polymorphs of $\mathrm{CaCO}_{3}$ are detected: vaterite and calcite. The diffraction peaks located at $2 \Theta=21.0^{\circ}, 24.9^{\circ}, 27.0^{\circ}, 32.8^{\circ}, 43.8^{\circ}$, and $50.0^{\circ}$ are assigned to the (004), (110), (112), (114), (300) and (118) planes of vaterite crystals, respectively, whereas the peaks at $2 \Theta=$ $29.4^{\circ}, 35.8^{\circ}$ and $48.6^{\circ}$ are assigned to the (104), (110) and (116) plane of calcite crystals, respectively. They are in good agreement with the reported values (PDF 33-0268 (vaterite) and PDF 05-0586 (calcite)). Moreover the (112), (110) and (114) diffraction peaks of vaterite are the strongest ones, indicating the preferential growth of these planes. Some traces of $\mathrm{NaCl}$ are noticed as well (PDF 05-0628).

To explain the formation of calcite/vaterite mixture it must be considered that vaterite is typically formed of amorphous $\mathrm{CaCO}_{3}$ (ACC) that is transferred to porous vaterite crystals through spherulitic growth, which is maintained as long as soluble ACC is present in the system. The size of vaterite phase increases as long as the ACC is consumed. The more stable calcite phase is formed with time, through the stepwise: ACC $\rightarrow$ 
(a)

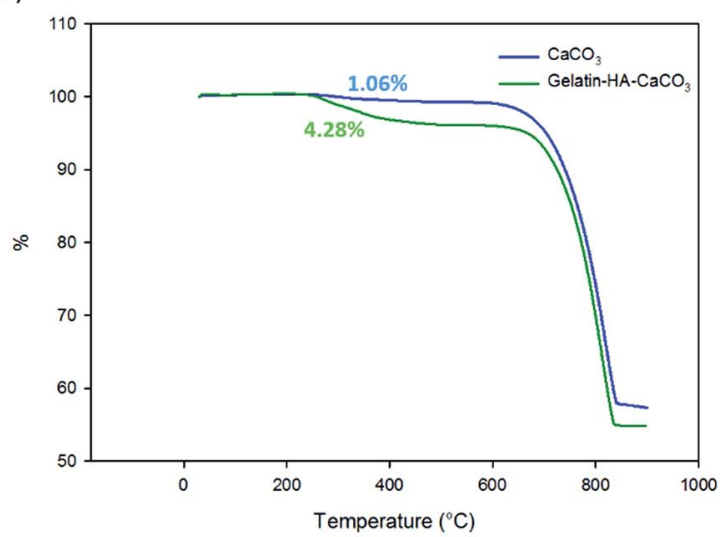

(b)

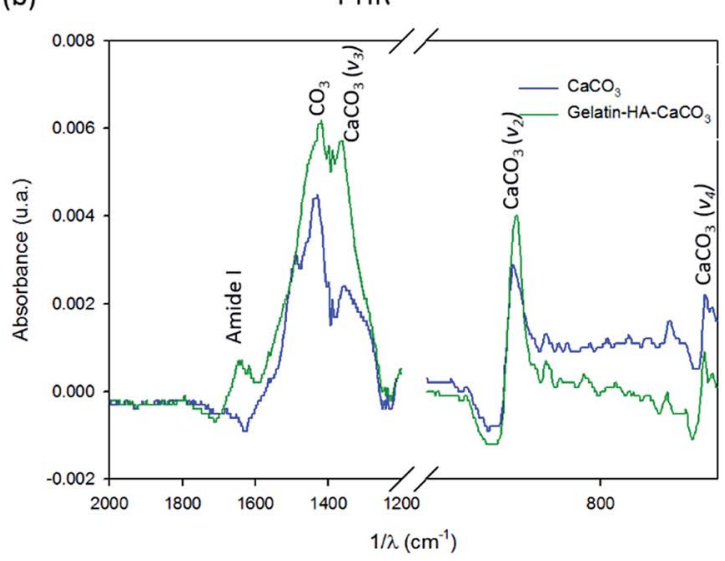

(c)

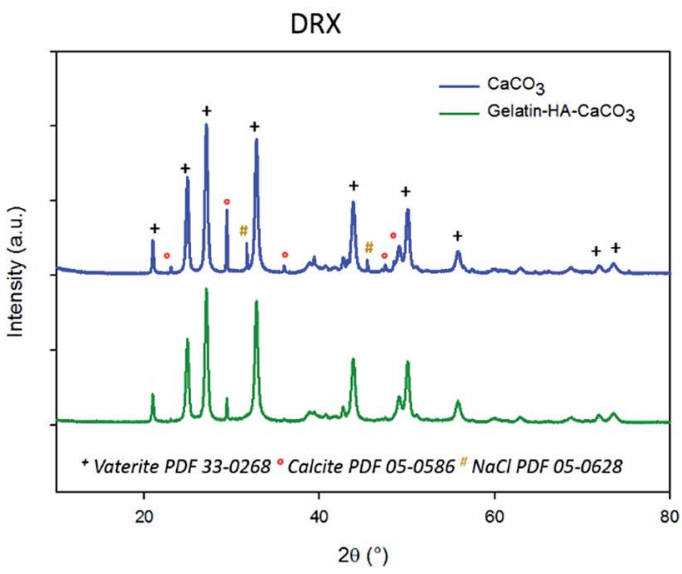

Fig. 2 Characterization of $\mathrm{CaCO}_{3}$ and gelatin- $\mathrm{HA}-\mathrm{CaCO}_{3}$ particles after crosslinking with transglutaminase by: (a) thermal gravimetric analysis (TGA), (b) Fourier transform infrared spectroscopy (FTIR) and (c) XDR spectra. ${ }^{+}$Vaterite (PDF 33-0268), e calcite (PDF-05-0586).

vaterite $\rightarrow$ calcite pathway. ${ }^{\mathbf{1 4}}$ However, the presence of some organic additives prevents the vaterite transformation to calcite, preserving the spherical morphology. Thus, the organic additives not only stabilizes vaterite phase, but also influences the particle size. For instance, some authors described the use of chondroitin sulfates and poly(sodium 4-styrene sulfate) (PSS) in the medium of $\mathrm{CaCO}_{3}$ precipitation in order to obtain a globular particle. ${ }^{9}$ In the present work, in order to prevent the formation of calcite, the formation of particles was performed in the presence of $2 \%$ gelatin. Without gelatin, the $\mathrm{CaCO}_{3}$ formation leads to the calcite precipitation (Fig. S1 $\dagger$ ).

The textural properties of the materials were analyzed by nitrogen adsorption/desorption and the obtained isotherms are depicted in Fig. 3a. The materials present a type III isotherm according to IUPAC classification and they are also characterized by a type III hysteresis characteristic of the presence of mesopores. BET analysis revealed a higher specific surface area (SSA) for gelatin-HA-CaCO ${ }_{3}$ microparticles $\left(46 \pm 1 \mathrm{~m}^{2} \mathrm{~g}^{-1}\right)$ than for $\mathrm{CaCO}_{3}$ microparticles $\left(15 \pm 0.1 \mathrm{~m}^{2} \mathrm{~g}^{-1}\right)$. This result may be explained by the diffusion and adsorption of gelatin in between the nanoparticles composing the $\mathrm{CaCO}_{3}$ microparticles and consequently the formation of additional voids leading to an increase in the specific surface area. A schematic representation of this process is illustrated in Fig. $3 \mathrm{~b}$. Additionally, the pore volume increases (from 0.10 to $0.25 \mathrm{~cm}^{3} \mathrm{~g}^{-1}$, Table 1) upon the gelatin penetration probably because $\mathrm{CaCO}_{3}$ nanoparticles are repulsed by the adsorbed gelatin.

The biodegradation of the particles was then studied. No calcium release was found from the gelatin-HA- $\mathrm{CaCO}_{3}$ microparticles at 1 day of immersion in the RPMI 1640 medium (Gibco) with $10 \%$ fetal bovine serum and $1 \%$ penicillin/ streptomycin at $37{ }^{\circ} \mathrm{C}$. However, from 1 to 7 days the release was progressive until $1.5 \mu \mathrm{g} \mathrm{mL} \mathrm{m}^{-1}$ (Fig. S2 $\dagger$ ).

The cells-microparticles system were obtained by alternate deposition of gelatin-HA- $\mathrm{CaCO}_{3}$ crosslinked microparticles dispersed in fibronectin (FN), ${ }^{15}$ NIH 3 T3 fibroblast and a second layer of microparticles/FN. As gelatin is soluble in aqueous

(a)

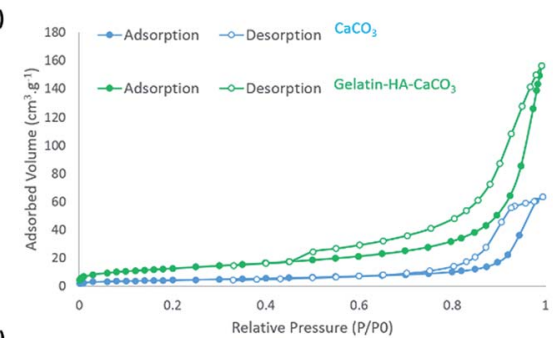

(b)

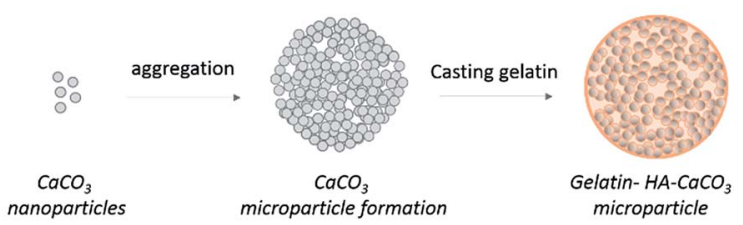

Fig. $3 \mathrm{~N}_{2}$ adsorption and desorption isotherms at $77 \mathrm{~K}$ of $\mathrm{CaCO}_{3}$ and gelatin- $\mathrm{HA}-\mathrm{CaCO}_{3}$ microparticles (a), and scheme of gelatin-HA$\mathrm{CaCO}_{3}$ microparticle formation (b).

Table 1 Textural properties of materials

\begin{tabular}{lll}
\hline $\mathrm{BJH}$ adsorption & Pore volume $\left(\mathrm{cm}^{3} \mathrm{~g}^{-1}\right)$ & $\mathrm{SSA}^{a}\left(\mathrm{~m}^{2} \mathrm{~g}^{-1}\right)$ \\
\hline $\mathrm{CaCO}_{3}$ & $0.10 \pm 0.01$ & $15 \pm 0.1$ \\
Gelatin-HA-CaCO $_{3}$ & $0.25 \pm 0.02$ & $46 \pm 1$
\end{tabular}

${ }^{a}$ Specific surface area. 
solution at $37{ }^{\circ} \mathrm{C}$, its use for biomedical applications rely on improvement of its mechanical and thermal stability by crosslinking. The crosslinking were done by enzymatically catalyzed reaction (transglutaminase ${ }^{\mathbf{1 6}}$ ). This enzyme catalyzes the formation of amide crosslinks from glutamine and primary amine functionalities from lysine. ${ }^{17}$ It appears to be one of the most suitable methods for biomedical indication due to its absence of toxicity combined to a high efficacy. It was observed that the system was not stable without the fibronectin, which explains its use as a ligand between the gelatin- $\mathrm{HA}-\mathrm{CaCO}_{3}$ microparticles, as it is a glycoprotein that binds to extracellular matrix components, such as collagen (gelatin).

Biocompatibility of the microparticles was assessed with NIH-3T3 fibroblast cells that were seeded with gelatin-HA$\mathrm{CaCO}_{3}$ crosslinked microparticles dispersed in (FN). The seeding efficacy was found more important $(t$-test, $P<0.05)$ for cells seeded on the particles than on the control (NIH-3T3 fibroblast cell cultured on 96 well plates with glass bottom), as shown in Fig. 4a. Compared to the control, the initial cell adhesion (4 hours after seeding) was an event basically driven by the presence of the extracellular matrix components present on the microparticles system: fibronectin, gelatin and HA.

Cell proliferation of $\mathrm{NHI}-3 \mathrm{~T} 3$ cells in gelatin-HA-CaCO microparticles scaffold was assessed during 7 days by following metabolic activity (Fig. 4b). It was shown that metabolic activity is increasing with time. After 1 day of culture, the fibroblasts cultured in the presence of the particles showed higher metabolic activity in comparison with the control ( $t$-test, $p<0.05)$. At days 3 and 7 no difference is recorded between the cells cultivated in gelatin- $\mathrm{HA}-\mathrm{CaCO}_{3}$ and on a control surface. This shows the gelatin- $\mathrm{HA}-\mathrm{CaCO}_{3}$ is suitable for in vitro cell culture.

Cell organization in gelatin- $\mathrm{HA}-\mathrm{CaCO}_{3}$ microparticles was assessed by CLSM as shown in Fig. 4c-e. The particles were labelled with HA-FITC (fluorescein isothiocyanate, green) and

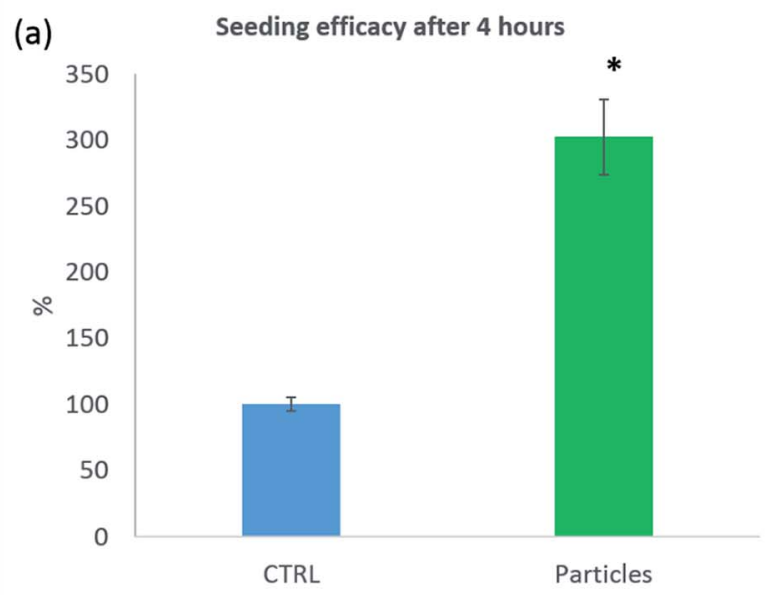

(b)

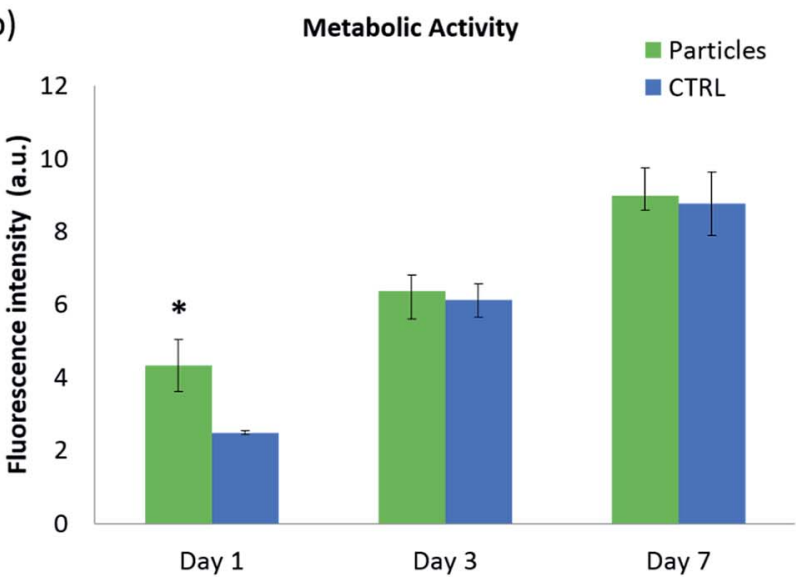

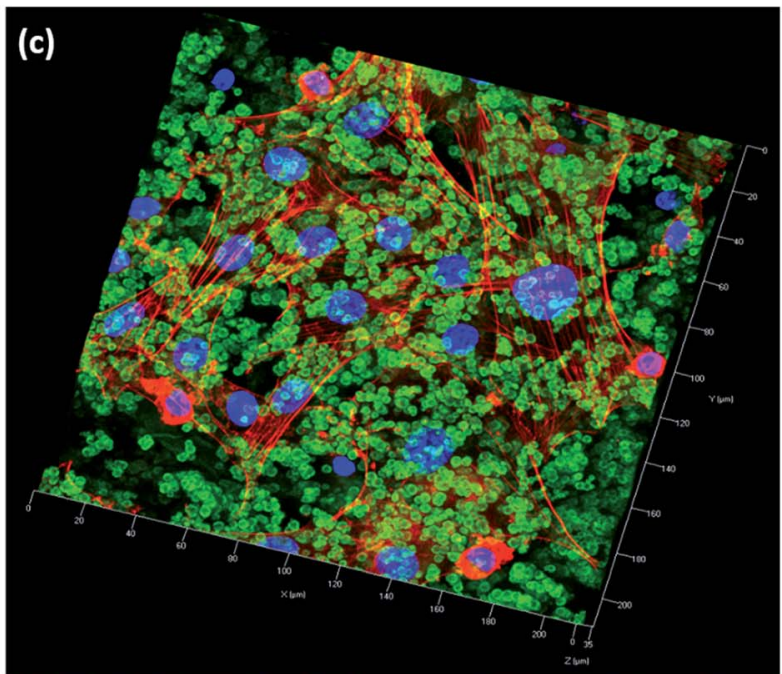

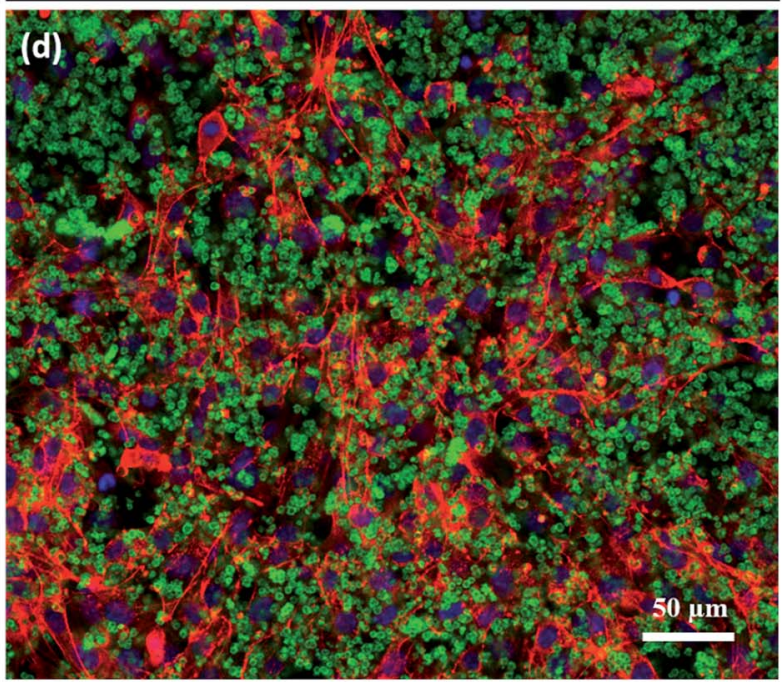

Fig. 4 Biological analysis of the system gelatin- $\mathrm{HA}-\mathrm{CaCO}_{3}$ microparticles in fibronectin solution and NIH $3 \mathrm{~T} 3$ fibroblast cells. (a) Seeding efficacy of NIH-3T3 fibroblast cells after 4 hours in culture, assessed by MTT test. (b) Metabolic activity of NIH 3T3 fibroblast after 1, 3 and 7 days of culture, assessed by Alamar Blue assay. * Significant difference between particles and control $(t$-test, $P<0.05)$. Confocal images of the after 3 days of culture: (c) 3D images and (d) 2D image. The particles are labelled in green (stained by HA-FITC) and the fibroblasts were visualized with Rho-phalloidin (red) and nuclei (blue). 
actin fibers and the nuclei of the fibroblasts were labelled with rhodamine phalloidin (red) and Hoechst (blue), respectively. Fig. 4c presents a 3D image of the final system. The cells seem to growth as a cell sheet between the two layers of microparticles, dispersed among the cells, without any obvious sign of invasion in surrounding layers, as shown in the $x y$ section (Fig. 4d). Those results provide a first approach to build predictive models of cellular self-assembly, as well as a simple biomimetic cell-culture platform to produce in a controlled way new tissues.

In summary, we described the design of a new system of hybrid microparticles mimicking the extracellular matrix. The results indicated that the obtained $\mathrm{CaCO}_{3}$ microparticles present a mesoporous aspect, which allow diffusion and adsorption of gelatin-HA. The presence of the extracellular matrix components, fibronectin, gelatin and HA on the microparticles are crucial for the initial cell adhesion. The method described in this study is easy to apply, versatile and could allow the buildup of any kind of targeted tissues. This first study offers wide versatility as microparticles and various sorts of cells could be alternatively deposited to produce well defined 3D architectures and finally any kind of targeted tissues.

\section{Acknowledgements}

This project has received funding from the European Union's Seventh Framework Programme for research, technological development and demonstration under grant agreement no. 60269 (IMMODGEL). We kindly thank Eric Mathieu for SEM images, Loic Vidal for TEM analysis, Gauthier Schrodj for TGA experiments and Jean-Marc Le Meins for XRD measurements.

\section{References}

1 B. Alberts, A. Johnson, J. Lewis, M. Raff, K. Roberts and P. Walter, Cell junctions, cell adhesion, and the extracellular matrix, 2002.
2 T. Owaki, T. Shimizu, M. Yamato and T. Okano, Biotechnol. J., 2014, 9(7), 904-914.

3 T. Osathanon, M. L. Linnes, R. M. Rajachar, B. D. Ratner, M. J. Somerman and C. M. Giachelli, Biomaterials, 2008, 29(30), 4091-4099.

4 H. Shokry, U. Vanamo, O. Wiltschka, J. Niinimäki, M. Lerche, K. Levon, M. Linden and C. Sahlgren, Nanoscale, 2015, 7(34), 14434-14443.

5 F. Caruso, R. A. Caruso and H. Möhwald, Science, 1998, 282(5391), 1111-1114.

6 E. Dolgin, Nat. Med., 2014, 20(1), 9-11.

7 Y. C. Lu, W. Song, D. An, B. J. Kim, R. Schwartz, M. Wu and M. Ma, J. Mater. Chem. B, 2015, 3(3), 353-360.

8 C. Wang, H. Liu, Q. Gao, X. Liu and Z. Tong, Carbohydr. Polym., 2008, 71(3), 476-480.

9 C. Du, J. Shi, J. Shi, L. Zhang and S. Cao, Mater. Sci. Eng., C, 2013, 33(7), 3745-3752.

10 A. Wang, Y. Cui, J. Li and J. van Hest, Adv. Funct. Mater., 2012, 22(13), 2673-2681.

11 H. Knopf-Marques, M. Pravda, L. Wolfova, V. Velebny, P. Schaaf, N. E. Vrana and P. Lavalle, Adv. Healthcare Mater., 2016, 5(18), 2841-2855.

12 Y. Ohyabu, H. Hatayama and S. Yunoki, J. Biosci. Bioeng., 2014, 118(1), 112-115.

13 D. B. Trushina, T. V. Bukreeva and M. N. Antipina, Cryst. Growth Des., 2016, 16(3), 1311-1319.

14 P. Bots, L. G. Benning, J. D. Rodriguez-Blanco, T. RoncalHerrero and S. Shaw, Cryst. Growth Des., 2012, 12(7), 38063814.

15 L. Poulouin, O. Gallet, M. Rouahi and J.-M. Imhoff, Protein Expression Purif., 1999, 17(1), 146-152.

16 M. K. McDermott, T. Chen, C. M. Williams, K. M. Markley and G. F. Payne, Biomacromolecules, 2004, 5(4), 1270-1279.

17 J. M. Orban, L. B. Wilson, J. A. Kofroth, M. S. El-Kurdi, T. M. Maul and D. A. Vorp, J. Biomed. Mater. Res., Part A, 2004, 68(4), 756-762. 